\title{
Aspects of linear Landau damping in discretized systems
}

Vasil Bratanov, Frank Jenko, David Hatch, and Stephan Brunner

Citation: Phys. Plasmas 20, 022108 (2013); doi: 10.1063/1.4792163

View online: http://dx.doi.org/10.1063/1.4792163

View Table of Contents: http://pop.aip.org/resource/1/PHPAEN/v20/i2

Published by the American Institute of Physics.

\section{Related Articles}

Shukla-Nambu-Salimullah potential with multi electron species in magnetoplasmas

Phys. Plasmas 20, 022107 (2013)

Discrete kinetic eigenmode spectra of electron plasma oscillations in weakly collisional plasma: A numerical study

Phys. Plasmas 20, 012125 (2013)

Non-planar ion-acoustic solitary waves and their head-on collision in a plasma with nonthermal electrons and warm adiabatic ions

Phys. Plasmas 20, 012122 (2013)

The incomplete plasma dispersion function: Properties and application to waves in bounded plasmas

Phys. Plasmas 20, 012118 (2013)

Effect of ion temperature on ion-acoustic solitary waves in a magnetized plasma in presence of superthermal electrons

Phys. Plasmas 20, 012306 (2013)

\section{Additional information on Phys. Plasmas}

Journal Homepage: http://pop.aip.org/

Journal Information: http://pop.aip.org/about/about_the_journal

Top downloads: http://pop.aip.org/features/most_downloaded

Information for Authors: http://pop.aip.org/authors

\section{ADVERTISEMENT}

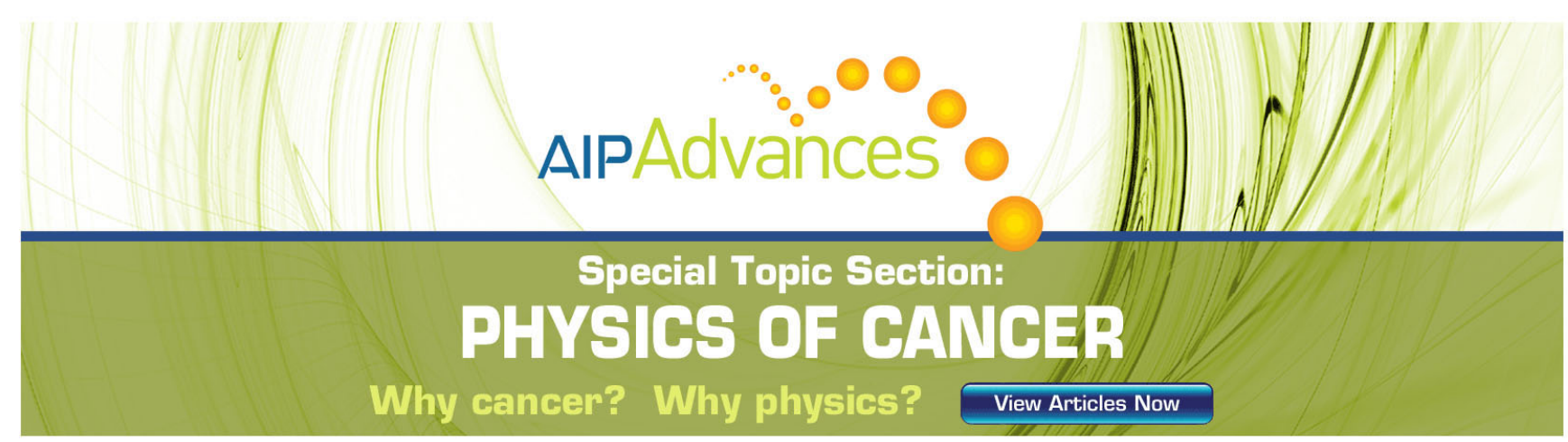




\title{
Aspects of linear Landau damping in discretized systems
}

\author{
Vasil Bratanov, ${ }^{1}$ Frank Jenko, ${ }^{1}$ David Hatch, ${ }^{1}$ and Stephan Brunner ${ }^{2}$ \\ ${ }^{1}$ Max-Planck-Institut für Plasmaphysik, EURATOM Association, 85748 Garching, Germany \\ ${ }^{2}$ Centre de Recherches en Physique des Plasmas, Ecole Polytechnique Fédérale de Lausanne (EPFL), \\ Association EURATOM - Confédération Suisse, CH-1015 Lausanne, Switzerland
}

(Received 20 November 2012; accepted 28 January 2013; published online 14 February 2013)

Basic linear eigenmode spectra for electrostatic Langmuir waves and drift-kinetic slab ion temperature gradient modes are examined in a series of scenarios. Collisions are modeled via a Lenard-Bernstein collision operator which fundamentally alters the linear spectrum even for infinitesimal collisionality [Ng et al., Phys. Rev. Lett. 83, 1974 (1999)]. A comparison between different discretization schemes reveals that a Hermite representation is superior for accurately resolving the spectra compared to a finite differences scheme using an equidistant velocity grid. Additionally, it is shown analytically that any even power of velocity space hyperdiffusion also produces a Case-Van Kampen spectrum which, in the limit of zero hyperdiffusivity, matches the collisionless Landau solutions. (C) 2013 American Institute of Physics. [http://dx.doi.org/10.1063/1.4792163]

\section{INTRODUCTION}

First predicted analytically ${ }^{1}$ and later observed experimentally, ${ }^{2}$ collisionless Landau damping is one of the most well known phenomena in plasma physics. The Landau solutions, all of which are damped for electrostatic Langmuir waves, arise after a Laplace transform with respect to time of the linearized governing equations and dominate the solution for large times. The advantage of such an approach is that it naturally includes the initial condition which is necessary when solving differential equations. However, Van Kampen searched for solutions with a time dependence of $\exp (i \omega t)$ and discovered a continuum of singular solutions that are marginally stable. ${ }^{3}$ It was demonstrated that the interplay of these modes leads to a damping of all moments of the distribution function, e.g., the electrostatic potential, given a smooth initial condition. An important conceptual point in the collisionless case that resolves this apparent contradiction is that the Landau approach applies to the electrostatic potential and not to the distribution function. Indeed, only moments of the distribution function with respect to velocity are damped but not the distribution function itself. Subsequent studies added collisions as a natural extension of the mathematical model. Using a simplified collision term of a Fokker-Planck type, Lenard and Bernstein found that the results of Landau do not change qualitatively in the presence of collisions, ${ }^{4}$ i.e., the Landau solutions change continuously with the collision frequency. Later, however, a similar investigation was conducted also for the Case-Van Kampen spectrum, and it was found numerically ${ }^{5}$ as well as analytically ${ }^{6}$ that collisions alter the Van Kampen spectrum completely; the continuum of marginally stable modes vanishes, and instead the spectrum consists of countably infinitely many eigenvalues that converge to the collisionless Landau solutions when the collision frequency tends to zero, i.e., collisions represent a singular perturbation of the system.

In this work we reexamine, analytically and numerically, several examples of basic kinetic eigenvalue problems, providing new insights into various aspects of the eigenmode spectra. The cause for the qualitative change of the spectrum that comes with introducing collisions is traced back to the second-order velocity derivative of the distribution function. In fact, this term alone is sufficient for reproducing the observed results. This we verify numerically and additionally show analytically that any even power of velocity space hyperdiffusion alters the spectrum in the same way, which is noteworthy considering that such terms are used extensively in computer simulations. Although the latter is performed mainly for numerical reasons, our analysis suggests that they capture more physical effects than might be expected at first sight.

For the collisional slab drift-kinetic model with ion temperature and density gradients, we shall focus our study on numerical approaches and compare different numerical schemes. We find that a truncated Hermite representation of the distribution function is in many ways superior to a finite differences approach on an equidistant velocity grid although the latter finds a wide application in numerical simulations.

One of the main motivations for this work was also the correct interpretation of the Case-Van Kampen-type spectral analyses, e.g., those performed with GENE, ${ }^{7}$ which has become an important task considering the various applications of linear eigenvalue computations. ${ }^{8-11}$ The results of this study are also expected to provide insights into the nonlinear effect of turbulence which is driven by the unstable linear eigenmodes studied here. Microturbulence and the associated transport in fusion plasmas frequently retain signatures of the underlying linear eigenmode spectra. ${ }^{12-16}$ Moreover, it has been shown recently that the interaction of unstable and stable eigenmodes at comparable scales facilitates turbulent saturation by providing an energy sink at the same scales as the energy drive. ${ }^{10,17-19}$ Thus, a careful study of both the unstable and stable parts of the linear eigenmode spectra is expected to lay the foundation for a deeper understanding of the saturation mechanisms which determine the level of heat transport in fusion plasmas.

The reminder of this paper is organized as follows. In Sec. II, we reproduce some known results, but in a mathematically more consistent way, by formulating the problem 
of plasma oscillations in the language of operator theory. This will illustrate the origin of the Van Kampen continuum and give a sufficient condition for its existence. Sec. III deals with the numerical implementation of the collisionless and collisional cases. We study the applicability of two different numerical schemes: finite differences on an equidistant grid in velocity space and a truncated Hermite representation. In Sec. IV we investigate analytically whether a diffusion term alone is sufficient to reproduce the qualitative change of the Case-Van Kampen spectrum observed numerically. The computation is generalized to an arbitrary even derivative with respect to velocity and conducted in a similar way as in Ref. 6. It is also noted that the solution of the initial value problem via a Laplace transform in time yields the same dispersion relation, i.e., collisional Case-Van Kampen and Landau solutions are the same when considering such dissipation terms. In Sec. V, the obtained results are summarized.

\section{MATHEMATICAL PROPERTIES OF THE LANGMUIR WAVE MODEL}

The starting point of our investigation is the model of one-dimensional collisionless electrostatic Langmuir waves with immobile ions distributed homogeneously in space. The dynamics are governed by the linearized Vlasov equation for the electron distribution function $g(z, v, t)$ combined with the Poisson equation. Space and time quantities are normalized to the Debye length, $\lambda_{D}$, and the electron plasma frequency, $\omega_{p e}$, defined as $\lambda_{D}=\sqrt{\varepsilon_{0} k_{B} T_{0} /\left(n_{0} e^{2}\right)}$ and $\omega_{p e}=\sqrt{n_{0} e^{2} /\left(m_{e} \varepsilon_{0}\right)}$, respectively. The thermal velocity we set as $v_{t h}=\lambda_{D} \omega_{p e}$. Using these units, we redefine further $g \rightarrow g n_{0} / v_{t h}$, $E \rightarrow k_{B} T_{0} E /\left(e \lambda_{D}\right), z \rightarrow z \lambda_{D}, t \rightarrow t / \omega_{p e}$, and $v \rightarrow v v_{t h}$, where $E$ denotes the electric field. The full distribution function is split into an equilibrium part $f_{0}(v)$ and a small perturbation $g_{1}(z, v, t)$ that is to be computed. Linearizing the Vlasov equation yields

$$
\frac{\partial g_{1}}{\partial t}+v \frac{\partial g_{1}}{\partial z}-E(z, t) \frac{\partial f_{0}}{\partial v}=0 ; \frac{\partial E}{\partial z}=-\int_{-\infty}^{+\infty} g_{1}(z, v, t) d v
$$

We shall also assume that the distribution is periodic in space with period $L$. Hence, it suffices to consider only the interval $z \in[0, L]$. Such a treatment suggests the use of a Fourier series representation in space defined by

$$
\hat{f}(k):=\frac{1}{L} \int_{0}^{L} f(z) e^{-i k z} d z \Rightarrow f(z)=\sum_{k} \hat{f}(k) e^{i k z},
$$

where $k$ takes discrete values given by $k=n 2 \pi / L$ and $n \in \mathbb{N}_{0}$. In what follows, we search for functions of the form $\hat{g}_{1}(k, v, t)=f(k, v, \omega) e^{-i \omega t}$, where $f(k, v, \omega)$ is determined by the equation

$$
\omega f(k, v, \omega)=k v f(k, v, \omega)-\frac{1}{k} \frac{\partial f_{0}}{\partial v} \int_{-\infty}^{+\infty} f\left(k, v^{\prime}, \omega\right) d v^{\prime} .
$$

This system can be viewed as an eigenvalue equation with $\omega$ as an eigenvalue and the right-hand side as a linear operator $A$ in velocity space acting on $f$, i.e.,

$$
(A f)(v):=k v f(v)+\psi(v) \int_{-\infty}^{+\infty} f\left(v^{\prime}\right) d v^{\prime}
$$

where $\psi(v)=-(1 / k)\left(\partial f_{0} / \partial v\right)$. For a proper eigenvalue equation one has to specify also the domain of definition of the operator $A$. An important observation is that the first part on the right-hand side of Eq. (4) is, up to the multiplicative factor $k$, the position operator from quantum mechanics for which one usually uses the Schwartz space $\mathcal{S}$ as the functional domain. We recall that $\mathcal{S}$ is the space of all $C^{\infty}$ functions which, as well as all their derivatives, decrease faster than any polynomial for large absolute values of the argument. In the explicit examples that shall be discussed in this paper, $\psi(v)$ has the form of a Gauss function multiplied by some polynomial, i.e., $\psi(v) \in \mathcal{S}$ and, therefore, the righthand side of Eq. (4) is well defined for all $f \in \mathcal{S}$.

$$
A: \mathcal{S} \subset L^{2}\left(\mathbb{R},\left(1+x^{2}\right) d x\right) \rightarrow \mathcal{S} \subset L^{2}\left(\mathbb{R},\left(1+x^{2}\right) d x\right),
$$

where $L^{2}\left(\mathbb{R},\left(1+x^{2}\right) d x\right)=: \mathcal{H}$ is the functional space of all functions over the real numbers that are square integrable with respect to the measure $\left(1+x^{2}\right) d x$. It is immediately clear that $\mathcal{H}$ is a Hilbert space with respect to the scalar product

$$
\langle f, g\rangle_{\mathcal{H}}:=\int_{-\infty}^{+\infty} \overline{f(v)}\left(1+v^{2}\right) g(v) d v
$$

where $\bar{f}$ stands for the complex conjugate of $f$. This turns $A$ into a densely defined operator, i.e., $A$ is defined on a functional space that is dense in a Hilbert space. In order to establish the connection to the physical problem one should elaborate on the physical relevance of the functional space $\mathcal{H}$. A reasonable condition is to demand that the particle density is always finite, i.e., $\int_{-\infty}^{+\infty} g(z, v, t) d v<\infty$ for all $t \in \mathbb{R}$ and $z \in[0, L]$. Since the one particle distribution function is per definition non-negative and the smallness condition $\left|g_{1}\right| \ll f_{0}$ applies for all $v \in \mathbb{R}$, it follows also that $g_{1}(z, v, t) \in L^{1}(\mathbb{R})$ with respect to $v$. Further, the perturbation $g_{1}(z, v, t)$ should have no singularities as a function of $z$ and $v$. From this and the previous considerations follows immediately that $f(k, v, \omega) \in L^{1}(\mathbb{R})$ with respect to $v$. Since all velocity moments of the distribution function have to be finite, it holds that $\lim _{v \rightarrow \pm \infty} v^{n} f(k, v, \omega) \rightarrow 0$ for all $n \in \mathbb{N}_{0}$, which together with the absence of singularities means that $f(k, v, \omega) \in L^{2}(\mathbb{R}, d x)$, i.e., $f(k, v, \omega) \in L^{1}(\mathbb{R}, d x) \cap L^{2}(\mathbb{R}, d x)$. The space $\mathcal{H}$ that we used is dense in $L^{1}(\mathbb{R}, d x) \cap L^{2}(\mathbb{R}, d x)$. The operator $A$ consists of two parts $A_{0}$ and $A_{1}$ defined as

$$
\left(A_{0} f\right)(v):=k v f(v), \quad\left(A_{1} f\right)(v):=\psi(v) \int_{-\infty}^{+\infty} f\left(v^{\prime}\right) d v^{\prime} .
$$

$A_{0}$ is essentially the position operator known from quantum mechanics which is self-adjoint on $\mathcal{S}$. It is also well known that the position operator has only an essential spectrum that consists of the whole real axis. An important point is that $A_{1}$ 
is bounded on $\mathcal{H},\left\|A_{1}\right\|_{\mathcal{H}} \leq \sqrt{\pi}\|\psi\|_{\mathcal{H}}<\infty$, which together with the fact that its range is finite dimensional implies that $A_{1}$ is compact on $\mathcal{H}$. According to a theorem of Weyl (p. 113, Corollary 2 in Ref. 20), in this case the essential spectrum of $A$ is the same as that of $A_{0}$, i.e., $\sigma_{e s s}(A)=\sigma_{e s s}\left(A_{1}\right)$. From this follows that every real number belongs to the spectrum of $A$, which is the result of Van Kampen. ${ }^{3}$ However, the theorem of Weyl does not exclude the possibility that there exist also complex values of $\omega$ that belong to the spectrum of $A$. One way to determine this is to find the resolvent operator of $A-\omega$. After a simple computation, one finds that it is given by

$$
\begin{aligned}
R_{A}(\omega)= & \frac{1}{k v-\omega}-\frac{\psi(v)}{k v-\omega} \frac{1}{\left(1+p \cdot v \cdot \int_{-\infty}^{+\infty} \frac{\psi\left(v^{\prime}\right)}{k v^{\prime}-\omega} d v^{\prime}\right)} \\
& \times p \cdot v \cdot \int_{-\infty}^{+\infty} \frac{d v^{\prime \prime}}{k v^{\prime \prime}-\omega}
\end{aligned}
$$

where the first term should be understood as a multiplication operator and the second one as an integral operator with $p . v$. denoting the principal value of the integral (this form of the resolvent operator has been given independently in Ref. 21 where a similar problem is discussed. However, the model of magnetized plasma presented there deviates considerably from the one studied here). Having the above form, one can easily verify that $R_{A}(\omega)(A-\omega)=(A-\omega) R_{A}(\omega)=i d$ on $\mathcal{H}$, which can be viewed as the definition of the resolvent operator and that $R_{A}(\omega)$ is bounded on $\mathcal{H}$ when both $\Im(\omega) \neq 0$ and $1+p \cdot v \cdot \int_{-\infty}^{+\infty} \frac{\psi\left(v^{\prime}\right)}{k v-\omega} d v \neq 0$ (here $i d$ and $\Im$ denote the identity operator and the imaginary part of a complex number, respectively). It is clear that $R_{A}(\omega)$ has poles at every real number. This gives again the continuous spectrum discovered by Van Kampen. However, $R_{A}(\omega)$ has poles also for frequencies which satisfy the relation

$$
1+p \cdot v \cdot \int_{-\infty}^{+\infty} \frac{\psi(v)}{k v-\omega_{0}} d v=0
$$

These solutions we shall call the discrete part of the Case-Van Kampen spectrum. We note here that, in the drift-kinetic system discussed in Sec. III, the discrete part of the collisionless Case-Van Kampen spectrum is connected to the unstable drift waves that arise in the case with background gradients. Thus, the mathematical foundation for a broader class of eigenmodes is already observed even in the simple electrostatic Langmuir wave system. For $\psi(v)$ a real valued function, the solutions of Eq. (9) are either real or appear in complex conjugate pairs. Considering the definition of the Landau contour, one should note that all unstable $(\Im(\omega)>0)$ Landau solutions coincide with the unstable Case-Van Kampen solutions. The eigenfunctions $f_{\omega}^{(V)}$ of $A$ have the form $f_{\omega}^{(V)}=-\frac{\psi(v)}{k v-\omega}$ $+k \delta(k v-\omega)\left(1+p \cdot v \cdot \int_{-\infty}^{+\infty} \frac{\psi\left(v^{\prime}\right)}{k v^{\prime}-\omega} d v^{\prime}\right)$ and are also given in Ref. 3. For $\omega$ real, $f_{\omega}^{(V)}(v)$ are distributions and belong neither to $L^{2}$ nor $L^{1}$. Therefore, in strict mathematical terms, real frequencies belong to the spectrum of $A$ but are not eigenvalues.
If $\Im\left(\omega_{0}\right) \neq 0$, then $f_{\omega_{0}}^{(V)} \in \mathcal{H}$, i.e., the discrete part of the spectrum of $A$ that does not lie on the real axis consists of mathematical eigenvalues. Using the explicit form of the eigenfunctions $f_{\omega}^{(V)}(v)$, one can verify that in contrast to the statement in Ref. 22, eigenfunctions corresponding to different frequencies are not orthogonal with respect to the scalar product

$$
\langle f, g\rangle_{f_{0}}=\int_{-\infty}^{+\infty} \frac{\overline{f(v)} g(v)}{f_{0}(v)} d v
$$

but, instead, for Langmuir waves we have

$$
\left\langle f_{\omega_{1}}^{(V)}, f_{\omega_{2}}^{(V)}\right\rangle_{f_{0}}=-\frac{1}{k^{2}} \text { if } \omega_{1} \neq \omega_{2} .
$$

According to the classical paper of Case, ${ }^{23}$ one should expect "a continuum of solutions for all real $\nu$ such that not simultaneously $\eta\left(\nu_{i}\right)=0=\lambda\left(\nu_{i}\right)$," where $\eta(v)$ denotes our $\psi(v)$, $\nu=\omega / k$ and $\lambda\left(\nu_{i}\right)$ is the left-hand side of Eq. (9). This, however, cannot be a condition for the existence of the continuous part of the spectrum because, as the theorem of Weyl shows, the addition of a compact operator $A_{1}$ to the selfadjoint $A_{0}$ does not change its continuous spectrum. The operator $A$ inherits the continuous part of its spectrum on the real axis from $A_{0}$ and since $\eta(v)$ is present only in $A_{1}$, no condition involving $\eta(v)$ can influence the existence of the continuum as long as $\|\eta(v)\|_{\mathcal{H}}<\infty$ which is fulfilled for every physically reasonable equilibrium distribution function. For real discrete $\nu_{i}$, the condition given in Ref. 23 reads $\eta\left(\nu_{i}\right)=\lambda\left(\nu_{i}\right)=0$. However, as we see from Eq. (8), it is not necessary that $\eta\left(\nu_{i}\right)=0$. To summarize, as long as $A_{1}$ is compact, there is a continuum of eigenvalues that covers the whole real axis. The discrete set of complex solutions satisfies the condition given by Eq. (9).

\section{NUMERICAL SCHEMES FOR DISCRETIZED SYSTEMS}

While one can extract some analytical results in the collisionless case, including collisions into the model makes it mathematically much more difficult. For obtaining results in this case, it is therefore necessary to approach the problem numerically which is the goal of this section. We also study a different model where background temperature and density gradients are taken into account. A numerical study, however, introduces the important question of which is the most appropriate discretization scheme to use. Here we compare the results obtained with a finite difference scheme on an equidistant velocity grid versus those achieved via a Hermite representation of the distribution function and conclude that the latter is in many aspects superior to the first.

\section{A. Collisionless Langmuir waves}

First, we consider an equidistant grid of $N$ points on the velocity axis that covers the interval $\left[-v_{\max }, v_{\max }\right]$. All functions of velocity turn into vectors in $\mathbb{R}^{N}$ and the operator in Eq. (4) becomes a matrix, $M$, the eigenvalues of which 
represent the Case-Van Kampen spectrum. The details of the numerical representation we use here are discussed in Ref. 24. In the discretized system, one can easily solve for the time evolution of the distribution function, $\vec{F}(t)$, which is given by $\vec{F}(t)=e^{-i M t} \cdot \vec{F}(t=0)$ and for smooth initial conditions leads to an oscillatory behavior of the perturbation in velocity space that gets more and more rapid with time. This is the well-known linear phase mixing which results in an exponential decay of all moments of the perturbation. Solving numerically for the eigenvalues of the matrix $M$, one concludes that they all lie on the real axis but are not equidistant and accumulate at the points corresponding to the real part of the two least damped Landau solutions. This leads to the question if all Case-Van Kampen eigenmodes are equally important for the numerical description of the system. The eigenvectors of $M$ form a complete basis in $\mathbb{R}^{N}$ and can be used as such to decompose any initial condition $\vec{F}_{0}$. When a Maxwellian initial condition is decomposed on the basis of linear eigenmodes, one observes that the modes having frequencies clustered near the real part of the frequency of the least damped Landau solutions possess the largest coefficients. However, if one constructs a reduced initial condition composed of only this subset of modes, the corresponding initial value computation does not reproduce the exponential decay associated with Landau damping. This suggests that the interplay of all modes with non-negligible coefficients is essential for obtaining the damping.

\section{B. Collisionless slab ion temperature gradient (ITG) modes}

As a natural next step, we extend our study to a driftkinetic slab ITG model. ${ }^{25,26}$ Electrons are assumed to be adiabatic and only the $\vec{E} \times \vec{B}$ drift is taken into account. We consider only electrostatic perturbations in a strong homogeneous magnetic field in the $z$ direction, $\vec{B}=B \vec{e}_{z}$, and presume a plasma in a local thermal equilibrium where temperature and density vary in the $x$ direction. The thermal ion velocity is defined in this case as $v_{t h, i}:=\sqrt{k_{B} T_{i}\left(x_{0}\right) / m_{i}}$, where $x_{0}$ stands for a reference position along $x, m$ is the particle mass, and the subscript $i$ stands for the ion particle species. We consider only one ion species with the charge $+e$. The time scales in this model are given by the gyrofrequency $\omega_{g}=e B / m_{i}$. One also defines the thermal ion gyroradius $\rho_{i}=v_{t h, i} / \omega_{g}$. After integrating the drift-kinetic equation over $v_{\perp}$, the resulting distribution function $g\left(\vec{r}, v_{\|}, t\right)=$ : $f_{0 i}\left(x, v_{\|}\right)+g_{1 i}\left(\vec{r}, v_{\|}, t\right)$ is normalized over $n_{0 i}\left(x_{0}\right) / v_{t h, i}$ and the electron potential $\varphi$ over $k_{B} T_{i}\left(x_{0}\right) / e$. For the temperature and density we adopt $T \rightarrow T / T\left(x_{0}\right)$ and $n_{0 i} \rightarrow n_{0 i} / n_{0 i}\left(x_{0}\right)$. The linearized equations describing the system in this case read

$$
\begin{gathered}
\frac{1}{\rho_{*}} \frac{\partial g_{1 i}}{\partial t}+v_{\|} \frac{\partial g_{1 i}}{\partial z}-\frac{\partial \varphi}{\partial y} \frac{\partial f_{0 i}}{\partial v_{\|}}-\frac{\partial \varphi}{\partial z} \frac{\partial f_{0 i}}{\partial x}=0 ; \\
\varphi=\tau \frac{T_{e}(x)}{n_{0 i}(x)} \int_{-\infty}^{+\infty} g_{1 i}\left(\vec{r}, v_{\|}, t\right) d v_{\|},
\end{gathered}
$$

where $\rho_{*}=\rho_{i} / R, \tau=T_{e}\left(x_{0}\right) / T_{i}\left(x_{0}\right)$ and $f_{0 i}$ and $g_{1 i}$ denote the equilibrium and perturbed ion distribution functions, respectively. Eq. (12b) represents the adiabatic response of the electrons and couples the electrostatic potential to the perturbed ion distribution function. Note that $x$ only appears as a parameter in Eqs. (12a) and (12b) and the equations are thus local in this direction. Without loss of generality one may thus reduce the study to the reference position $x=x_{0}$ which is equivalent to setting $k_{x}=0$. In Eqs. (12a) and (12b) we have normalized $x$ and $y$ with respect to the microscopic scale $\rho_{i}$ and $z$ with respect to the macroscopic scale $R$ which characterizes the variation of temperature and density in the $x$ direction. For the temperature and density gradients we use the local approximation

$$
\frac{1}{T_{i}} \frac{d T_{i}}{d x}=:-\frac{1}{L_{T}}=\text { const } ; \quad \frac{1}{n_{0 i}} \frac{d n_{0 i}}{d x}=:-\frac{1}{L_{d}}=\text { const. }
$$

$L_{T}$ and $L_{d}$ we shall refer to as gradient lengths which are normalized over $R$. After a Fourier transformation with respect to $y$ and $z$, using the assumption that the perturbation of the ion distribution function is proportional to $e^{-i \omega t}$, one arrives at the eigenvalue equation

$$
\frac{\omega}{\rho_{*}} f_{1 i}=k_{\|} v_{\|} f_{1 i}-\tau \alpha\left(v_{\|}\right) f_{0 i}\left(v_{\|}\right) \int_{-\infty}^{+\infty} f_{1 i}\left(x, k_{y}, k_{\|}, v_{\|}^{\prime}, \omega\right) d v_{\|}^{\prime}
$$

where $\alpha\left(v_{\|}\right)=k_{y} /\left(2 L_{T}\right)-k_{y} / L_{d}-k_{\|} v_{\|}-k_{y} v_{\|}^{2} /\left(2 L_{T}\right), f_{0 i}\left(v_{\|}\right)$ $=\exp \left(-v_{\|}^{2} / 2\right) / \sqrt{2 \pi}, \quad$ and $\quad g_{1 i}\left(k_{y}, k_{\|}, v_{\|}, t\right)=: f_{1 i}\left(k_{y}, k_{\|}\right.$, $\left.v_{\|}, \omega\right) e^{-i \omega t}$. The discretization procedure is the same as for Langmuir waves. The spectra that the corresponding matrix produces are shown in Fig. 1 where the blue asterisks and green crosses denote the continuous and the discrete part of the Case-Van Kampen spectrum, respectively. The latter is defined by Eq. (9). Because of the numerical discretization scheme the asterisks are contained on a finite interval in the $\omega$-plane that corresponds to the interval $\left[-v_{\max }, v_{\max }\right]$ in velocity space. Those discrete eigenvalues that stand out from the continuum part are computed directly by the numerical eigenvalue solver together with the blue asterisks. For the green crosses that are embedded into the continuum we used approximate solutions of Eq. (9). For comparison, the collisionless Landau solutions are also plotted (red circles). They solve the analytical dispersion relation

$$
\begin{aligned}
1 & +\frac{1}{\tau}+\frac{k_{y}}{2 k_{\|}^{2} \rho_{*} L_{T}} \omega+Z\left(\frac{\omega}{\sqrt{2} k_{\|} \rho_{*}}\right) \cdot\left(\frac{k_{y}}{2 \sqrt{2} k_{\|}^{3} \rho_{*}^{2} L_{T}} \omega^{2}\right. \\
+ & \left.\frac{\omega}{\sqrt{2} k_{\|} \rho_{*}}+\frac{k_{y}}{\sqrt{2} k_{\|} L_{n}}-\frac{k_{y}}{2 \sqrt{2} k_{\|} L_{T}}\right)=0
\end{aligned}
$$

that arises after applying a Laplace transform with respect to time on Eq. (14). $Z$ denotes the plasma dispersion function as defined in Ref. 27.

It is evident that the Case-Van Kampen spectra of driftkinetic slab ITG and Langmuir waves display a great deal of similarity. In both cases one observes the kinetic modes on the real axis that result from the first part of the linear operator. For some parameters there are also (possibly complex) eigenvalues present that result from the second part of the 
(a) $L_{T}=5, L_{d}=20$

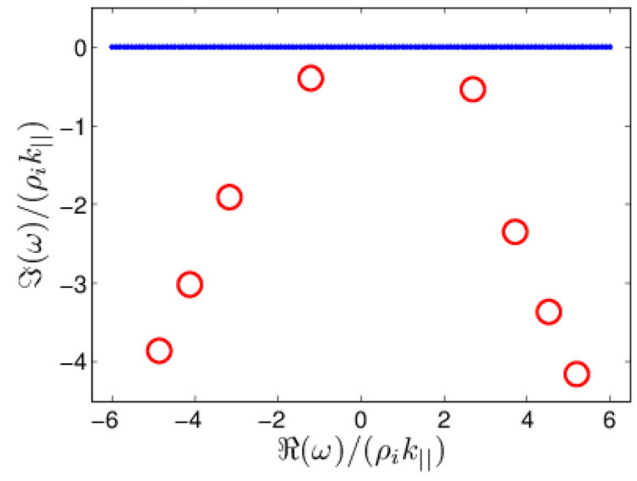

(c) $L_{T}=1, L_{d}=4$

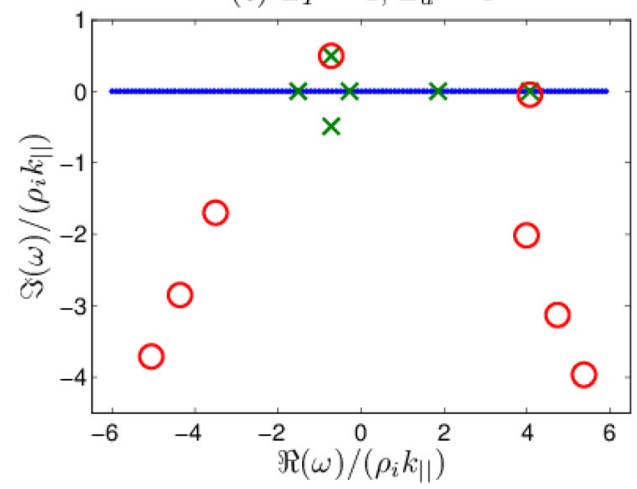

(b) $L_{T}=2.5, L_{d}=10$

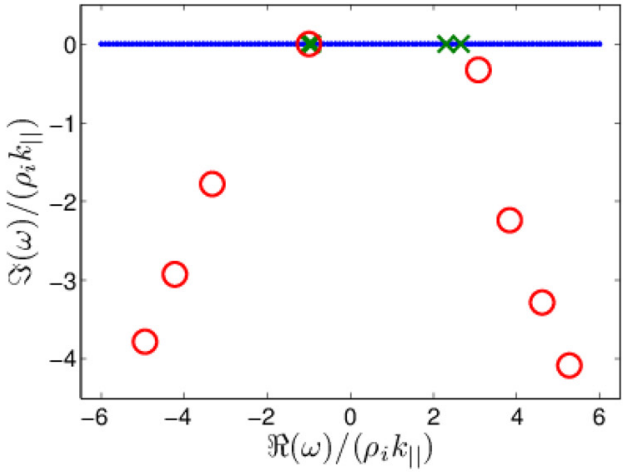

(d) $L_{T}=0.25, L_{d}=1$

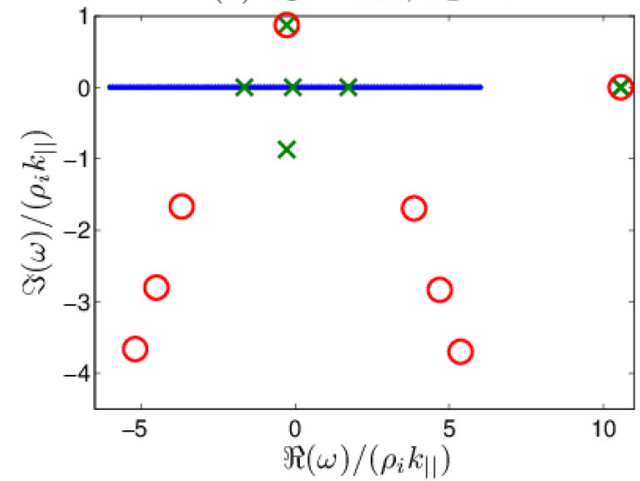

FIG. 1. Evolution of the discrete collisionless Case-Van Kampen eigenmodes (green crosses) and the collisionless Landau solutions (red circles) with respect to the temperature and density gradient lengths. For large gradients the ion acoustic mode emerges which is almost marginally stable and practically coincides with one of the discrete CaseVan Kampen eigenmodes. The unstable discrete Case-Van Kampen modes match the unstable Landau solutions and the blue asterisks represent the continuum part of the Van Kampen spectrum. $k_{y}=0.3, k_{\|}=0.03$. resolvent operator in Eq. (8) and that we call the discrete Case-Van Kampen spectrum. To this set belongs also the isolated mode on the right which is very close to one of the Landau solutions. The latter is due to the fact that for $|\Re(\omega)| \gg|\Im(\omega)|$, the plasma distribution function equals nearly the principle value integral for the corresponding complex frequency. We shall refer to this mode as an ion acoustic mode. This association is also mathematically motivated. It is well known that ion sound waves due to density gradients have frequencies that are proportional to $k_{y} /\left(k_{\|} L_{d}\right)$. Using the drift-kinetic Landau dispersion relation, one can derive a differential equation that governs the movement of the Landau solutions in the complex frequency plane with respect to any of the parameters. For $1 / L_{T}=0$ it is immediately seen that the frequency of solutions with negligible damping/growth rate also scales as $k_{y}$ with the proportionality constant of $1 /\left(k_{\|} L_{d}\right)$.

In Fig. 1, one can also follow the movement of the eigenvalues and the Landau solutions in the complex plane resulting from varying the gradient lengths. Correspondingly, not only the position but also the number of existing discrete eigenvalues change. For one set of parameters given by the condition $k_{y}^{2} / k_{\|}^{2}=4(1+\tau) L_{T}^{2} L_{d} /\left(\tau^{2}\left(L_{d}-2 L_{T}\right)\right)$, one of the discrete eigenvalues on the real axis coincides with one of the Landau solutions that by further increment of the temperature gradient becomes the instability. For $k_{y}=10 k_{\|}$ and $L_{d}=10$ this is realized for $L_{T}=2.5$ and shown in Fig. 1(b). This condition also means that for given gradient lengths, there are always $k_{y}$ and $k_{\|}$values such that the ratio $k_{y} / k_{\|}$fulfills the upper condition, and there is an undamped Landau solution with the same frequency as one of the discrete Case-Van Kampen eigenvalues. In conclusion, despite the singular nature of the collisionless Case-Van Kampen modes with real frequency, the discrete Case-Van Kampen spectrum shows an interesting connection to that part of the Landau solutions that has a clear physical interpretation, namely, the instability and the drift wave.

It is noteworthy that the discrete Case-Van Kampen eigenvalues have the same dispersion relation (Eq. (9)) as the nonlinear undamped Bernstein-Greene-Kruskal modes when the amplitude of the latter tends to zero. ${ }^{28}$

\section{Comparison between different discretization schemes for the collisional case}

Up to now, we discussed only collisionless systems. One could argue that such a model is a good approximation of hot fusion plasmas since the collision frequency in such plasmas is small. However, it has been shown numerically ${ }^{5}$ and later proved analytically ${ }^{6}$ that for any non-zero collision frequency, collisions are important even for a correct qualitative description of the system (at least, if collisions are modeled via the Lenard-Bernstein collision operator first introduced in Ref. 4). In that case, the Case-Van Kampen spectrum becomes fully discrete, and it is the same as the corresponding Landau solutions.

$\mathrm{Ng}$ et al. ${ }^{5}$ use a Hermite representation in order to compute the Case-Van Kampen spectrum. Our first goal is to investigate if the same result can be obtained by using finite differences on an equidistant grid in velocity space which is the most common one used in numerical studies, e.g., in GENE. Later, we will also determine which part of the Lenard-Bernstein collision operator is responsible for the qualitative change of the Case-Van Kampen spectrum. In 
this part we shall focus on the drift-kinetic slab ITG model, since it is more relevant for our future work. However, the statements that we are going to make about the usefulness of different discretization schemes apply in the same manner also to Langmuir waves. Modifying Eq. (14) by a LenardBernstein collision operator formulated in the parallel velocity direction leads to

$$
\begin{aligned}
\frac{\omega}{\rho_{*}} f_{1 i}= & k_{\|} v_{\|} f_{1 i}-\tau \alpha\left(v_{\|}\right) f_{0 i}\left(v_{\|}\right) \int_{-\infty}^{+\infty} f_{1 i}\left(\ldots, v_{\|}^{\prime}, \ldots\right) d v_{\|}^{\prime} \\
& +i \frac{\nu}{\rho_{*}}\left(f_{1 i}+v_{\|} \frac{\partial f_{1 i}}{\partial v_{\|}}+\frac{\partial^{2} f_{1 i}}{\partial v_{\|}^{2}}\right),
\end{aligned}
$$

where $\nu$ denotes the collision frequency normalized over the gyro-frequency $\omega_{g}$. Derivatives are modeled numerically by centered finite difference schemes defined as

$$
\frac{\partial f_{1 i}}{\partial v_{\|}} \rightarrow \frac{1}{2 \Delta v_{\|}}\left(X_{+1}-X_{-1}\right) \cdot \vec{F} ; \frac{\partial^{2} f_{1 i}}{\partial v_{\|}^{2}} \rightarrow \frac{1}{\Delta v_{\|}^{2}}\left(X_{+1}-2 I+X_{-1}\right) \cdot \vec{F},
$$

where $X_{-1}=X_{+1}^{T}$ and the elements of the matrix $X_{+1}$ are given by $\left(X_{+1}\right)_{i, j}=\delta_{i+1, j}$. For the Hermite representation, the expansion $f_{1 i}\left(k_{y}, k_{\|}, v_{\|}, \omega\right)=\sum_{n=0}^{\infty} a_{n}\left(k_{y}, k_{\|}, \omega\right) H_{n}$ $\left(v_{\|} / \sqrt{2}\right) e^{-v_{\|}^{2} / 2}$ is used where $H_{n}(x)=(-1)^{n}\left(2^{n} n ! \sqrt{\pi}\right)^{-1 / 2}$ $e^{x^{2}} d^{n} e^{-x^{2}} / d x^{n}$. With these definitions, the function $H_{n}\left(v_{\|} / \sqrt{2}\right) e^{-v_{\|}^{2} / 2}$ is an eigenfunction of the collision operator in Eq. (16) with the eigenvalue $-n$, and the integral of $f_{1 i}\left(k_{y}, k_{\|}, v_{\|}, \omega\right)$ over $v_{\|}$reduces to $\sqrt{2} \sqrt[4]{\pi} \mathrm{a}_{0}\left(k_{\mathrm{y}}, k_{\|}, \omega\right)$. The eigenfrequencies $\omega$ in the Hermite representation are given by a recurrence relation for the coefficients $a_{n}$ that reads

$$
\begin{aligned}
\frac{\omega}{k_{\|} \rho_{*}} a_{n}= & \frac{\tau k_{y}}{\sqrt{2} L_{T} k_{\|}} \delta_{2 n} a_{n-2}+\left(\sqrt{n}+\tau \delta_{1 n}\right) a_{n-1} \\
& +\left(\frac{\tau k_{y}}{L_{d} k_{\|}} \delta_{0 n}-\frac{i n \nu}{k_{\|} \rho_{*}}\right) a_{n}+\sqrt{n+1} a_{n+1}
\end{aligned}
$$

and is derived in the same way as the analogous recurrence relation in Ref. 5. The collisional Landau solutions that arise in our slab ITG model are determined by the dispersion relation

$$
\begin{aligned}
1+ & \frac{1}{\tau}+\frac{k_{y}}{2 \rho_{*} k_{\|}^{2} L_{T}} \omega+i \frac{k_{y}}{2 \rho_{*} k_{\|}^{2} L_{T}} \nu \\
& +i \exp \left(\frac{\rho_{*}^{2} k_{\|}^{2}}{\nu^{2}}\right) \cdot\left(\frac{\nu}{\rho_{*} k_{\|}}\right)^{2 \rho_{*}^{2} k_{\|}^{2} / \nu^{2}-2 i \omega / \nu-1} \\
& \times \gamma\left(\frac{\rho_{*}^{2} k_{\|}^{2}}{\nu^{2}}-i \frac{\omega}{\nu}, \frac{\rho_{*}^{2} k_{\|}^{2}}{\nu^{2}}\right) \cdot\left(\frac{k_{y}}{2 \rho_{*}^{2} k_{\|}^{3} L_{T}} \omega^{2}+\frac{\omega}{\rho_{*} k_{\|}}\right. \\
& \left.+\frac{k_{y}}{k_{\|}}\left(\frac{1}{L_{d}}-\frac{1}{2 L_{T}}\right)+i \frac{k_{y}}{2 \rho_{*}^{2} k_{\|}^{3} L_{T}} \nu \omega\right)=0
\end{aligned}
$$

where $\gamma$ denotes the incomplete gamma function as defined in Ref. 29.

The upper part of the spectra that one obtains this way for different collision frequencies is shown in Fig. 2 where the Case-Van Kampen modes (blue crosses) have been computed numerically with different discretization schemes and the Landau modes are the solutions of Eq. (15) (collisionless case, red circles) and Eq. (19) (collisional case, black (a) $\nu=0.1 \sqrt{2} \rho_{i} k_{\|} ;$velocity discretization

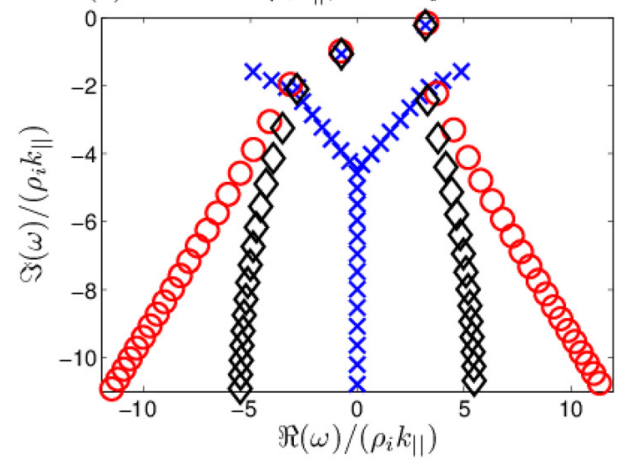

(c) $\nu=0.2 \sqrt{2} \rho_{i} k_{\|}$; velocity discretization

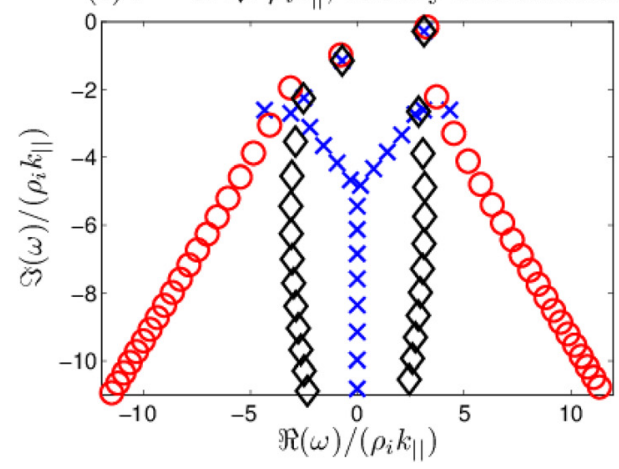

(b) $\nu=0.1 \sqrt{2} \rho_{i} k_{\|}$; Hermite representation

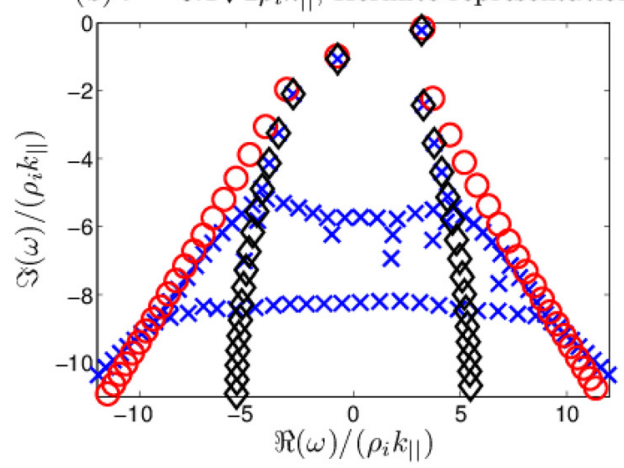

(d) $\nu=0.2 \sqrt{2} \rho_{i} k_{\|}$; Hermite representation

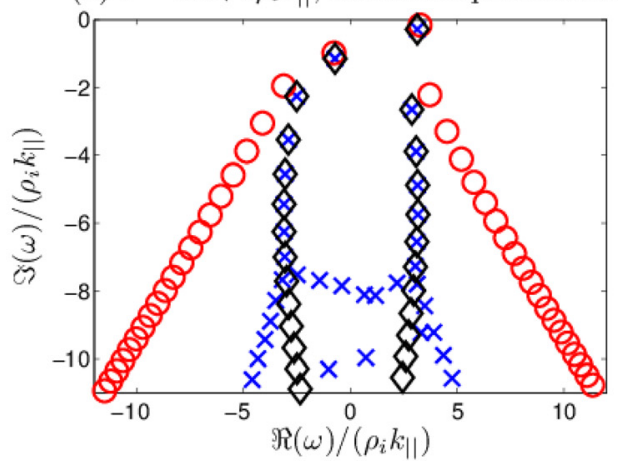

FIG. 2. Collisional Van Kampen spectrum (blue crosses) for two collision frequencies and different discretization schemes; red circles: collisionless Landau solutions; black diamonds: corresponding collisional Landau solutions; $k_{y}=0.3, k_{\|}=0.03$. The number of velocity grid points used equals 128 and is the same as the number of polynomials in the truncated Hermite scheme. 
diamonds). For a view on the whole spectra one can consult Ref. 24 where also the question of numerical convergence is discussed. Here, relatively large gradient lengths have been chosen such that there are only damped solutions. By increasing the temperature gradient, the left one of the two isolated modes becomes unstable and the right one moves further to the right while its damping rate decreases. An increase of the density gradient makes the right mode practically marginally stable and increases its frequency even further such that it becomes the ion acoustic mode. Performing the limit $\nu \rightarrow 0$, smaller structures in velocity space develop, which requires that the resolution in velocity space increases in order that the spectrum converges. In case of the finite difference approach, the form of the converged spectrum can be obtained by increasing the number of points on the interval $\left[-v_{\max }, v_{\max }\right]$ while keeping the collision frequency fixed. With this discretization scheme, it is observed that for small collision frequency only two of the Case-Van Kampen modes agree with the corresponding Landau solutions. Even a velocity resolution of nearly 32000 points could not produce more pairs of Case-Van Kampen modes which lie in the vicinity the Landau solutions. Another issue concerning the finite difference scheme is the fact that it produces a large number of numerical, i.e., non-physical, modes that have a rather small damping rate. For some values of the parameters, a subset of those numerical modes can be less damped even than the least damped Landau solutions. An initial value computation in this case shows, however, that the electric field still decreases with the rate of the least damped Landau solution, i.e., the interplay of those modes is such that they do not change the expected behavior of physical quantities like the electric field or the electrostatic potential. This is also the main reason for calling those modes nonphysical.

In Ref. 24 we observed numerical recurrence in the initial value simulation for both collisionless Langmuir waves and slab ITG modes. Recurrence also manifests itself clearly when using a collision operator of a Krook-type. However, with the Lenard-Bernstein collision operator the recurrence is eliminated, which represents another advantage since such a non-physical phenomenon is undesirable for computer simulations.

Figures 2(b) and 2(d) display again the Case-Van Kampen spectrum for collisional slab ITG modes, but this time obtained via the Hermite representation where the number of Hermite polynomials used equals the number of velocity points in Figures 2(a) and 2(c). The Hermite representation was introduced already in Ref. 30 for the study of experimental measurements of ion distribution functions which also motivated the theoretical studies that followed. In contrast to matching backward and forward recurrence relations for every eigenvalue in the case of small collision frequency as performed by $\mathrm{Ng}$ et al., ${ }^{5}$ we simply truncate the series at some finite order. This leads to a simple matrix eigenvalue equation which is readily solved numerically and yields good results for the less damped eigenmodes.

The comparison with the corresponding collisional Landau solutions shows a very good agreement in the upper part of the complex $\omega$-plane where there is a matching of many
Landau solutions with Case-Van Kampen eigenvalues. Nonphysical modes exist in this case only under the matched solutions which is not critical since these modes are strongly damped. The level at which the matching stops and only the numerical modes are present shifts upwards when collision frequency decreases and above a certain value of $N$ (nearly 100 ) is practically not influenced by $N$ any more. This implies that there exists a lower limit on the collision frequency below which it is impossible to properly resolve the eigenvalue spectrum with standard numerical methods. Fig. 2 illustrates that, first, for a fixed collision frequency, the Hermite representation produces more matchings of Landau modes than the finite differences and, second, increasing $\nu$ leads to a much greater improvement in the Hermite case than in the case of the finite difference scheme on an equidistant velocity grid. Note also that for a certain set of parameters, some of the non-physical modes can move so close to the real axis that there are no matchings any more and the whole Case-Van Kampen spectrum consists of numerical modes some of which are less damped than the least damped Landau solutions. An initial value computation in this case however still leads to electric field and electrostatic potential that decay with the damping rate of the least damped Landau solution and again no prominent numerical recurrence is observed.

The next step in our investigation is to determine which term in the collision operator is responsible for the qualitative change observed in the spectrum. As discussed more thoroughly in Ref. 24, the unexpected change comes from the second derivative of $f_{1 i}$ with respect to the parallel velocity. By leaving only this part of the collision operator and removing the other two, one obtains with the velocity discretization the same type of spectrum as shown in Figures 2(a) and 2(c). The same applies also to any derivative of an even power of the distribution function with respect to velocity. We shall elaborate on this and specify our statements in a more mathematical fashion in Sec. IV. While the velocity discretization appears to be rather robust regarding different collision operators, this is not the case with the Hermite representation since its advantage in the case of the Lenard-Bernstein operator, $\mathcal{C}_{L}$, is that the functions $b_{n}\left(v_{\|}\right)$ $:=H_{n}\left(v_{\|} / \sqrt{2}\right) e^{-v_{\|}^{2} / 2}$ used as a functional basis are the eigenfunctions of this operator, i.e., $\left(\mathcal{C}_{L} b_{n}\right)\left(v_{\|}\right)=-n b_{n}\left(v_{\|}\right)$. A substitution of $\mathcal{C}_{L}$ in this case with a second derivative with respect to $v_{\|}$alone yields entirely different and non-physical results. This can be easily deduced from the equations since now the Hermite functions are not eigenfunctions of the operator but instead $\partial^{n} b_{m} / \partial v_{\|}^{n} \propto \sqrt{(m+1) \ldots(m+n)} b_{m+n}$ which corresponds to a matrix the spectrum of which is $\sigma=\{0\}$ and not $\{-n\}_{n \in \mathbb{N}_{0}}$ as for $\mathcal{C}_{L}$. Therefore, in the Hermite representation one should not use hyperdiffusion-like terms as a simple dissipation source. A possible analogue would be $-\left(-\mathcal{C}_{L}\right)^{n}, n \in \mathbb{N}$, as suggested by Parker. ${ }^{31}$

\section{ROLE OF HYPERDIFFUSION TERMS}

Next, we investigate analytically the effect of hyperdiffusion-like terms in the linearized system of equations in both the case of Langmuir waves and slab ITG 
modes. A similar problem has been discussed in Ref. 6 where the analysis is performed for the Lenard-Bernstein collision operator which can be formulated in one dimension. In numerical computations, one often makes use of a socalled hyperdiffusion term which is proportional to the 4th derivative of the particle distribution function with respect to velocity. Such a term is also called numerical dissipation, and for this purpose one can use any even derivative of the distribution function in velocity space. ${ }^{32}$

\section{A. Langmuir waves}

For a better understanding of the problem, we first consider one-dimensional electrostatic Langmuir waves and use the same notation as in Refs. 5 and 6 according to which $\Omega:=\omega /(\sqrt{2} k), u:=v / \sqrt{2}$. This will facilitate comparison with the results obtained there. Thus, the linearized Boltzmann equation reads

$$
u g(u)-\Omega g(u)-\eta(u) \int_{-\infty}^{+\infty} g\left(u^{\prime}\right) d u^{\prime}=i \varepsilon\left(i^{n} \frac{\partial^{n} g}{\partial u^{n}}\right)
$$

where $\eta(u)=-\alpha u e^{-u^{2}} / \sqrt{\pi}$ with $\alpha=1 / k^{2} \in \mathbb{R}_{+}$, the term on the right-hand side in the brackets produces dissipation for every even number $n$, and $\varepsilon$ is some small positive parameter which we shall refer to as collision frequency. $g(u)$ denotes here the same quantity as $f(k, v, \omega)$ in Eq. (3) where for the ease of notation we have suppressed the arguments $k$ and $\omega$. In order to solve this equation, we perform a Fourier transform with respect to velocity

$$
G(p):=\frac{1}{\sqrt{2 \pi}} \int_{-\infty}^{+\infty} g(u) e^{i p u} d u \Rightarrow g(u)=\frac{1}{\sqrt{2 \pi}} \int_{-\infty}^{+\infty} G(p) e^{-i p u} d p
$$

With this definition, Eq. (20) becomes

$$
\frac{d G}{d p}+\left(\varepsilon p^{n}-i \Omega\right) G(p)=\frac{\alpha}{2} G(0) p e^{-p^{2} / 4} .
$$

We have written the equation as an ordinary differential equation because there are only derivatives with respect to $p$ and the other variables can be regarded as parameters. The solution of the above equation is

$$
\begin{aligned}
G(p)= & \frac{\alpha}{2} G(0) \exp \left(-\frac{\varepsilon}{n+1} p^{n+1}+i \Omega p\right) \\
& \times \int_{a}^{p} x \exp \left(\frac{\varepsilon}{n+1} x^{n+1}-x^{2} / 4-i \Omega x\right) d x \\
& +C \exp \left(-\frac{\varepsilon}{n+1} p^{n+1}+i \Omega p\right)
\end{aligned}
$$

where $a$ and $C$ are some constants. At this point, it might look surprising that the general solution of an ordinary firstorder differential equation depends on two constants instead of only one. However, one should note that the two constants are not independent from each other. We shall see that demanding that $G(p)$ is absolutely integrable determines both constants. This condition also assures that $g(u)$ is a continuous function. Actually, our analysis will show that $G(p)$ is even a Schwartz function which automatically implies the same for $g(u)$. Since $n$ is even, the second term in the solution diverges as $\exp \left(-p^{n+1}\right)$ when $p \rightarrow-\infty$. Thus, $C$ has to be zero in order that the inverse Fourier transform of $G(p)$ exists. This, however, is not a sufficient condition. It is also necessary that the first term in Eq. (23) goes to zero rapidly enough when $p \rightarrow-\infty$. This can be achieved by demanding that $a=-\infty$. This choice is unique because there is only one function that solves Eq. (22) and does not belong to the kernel of the operator. With the above values for the constants $a$ and $C G(p)$ goes to zero rapidly enough for $p \rightarrow-\infty$ such that it is absolutely integrable for large negative values of the argument. Next, we focus on the case $p \rightarrow+\infty$. This limit is slightly more problematic because the integral in Eq. (23) diverges when $p \rightarrow+\infty$. On the other hand, the absolute value of the prefactor in front of the integral goes rapidly to zero when $p$ increases. In order to clarify this limit, we estimate $|G(p)|$ from above as follows:

$$
\begin{aligned}
|G(p)|= & \frac{\alpha}{2}|G(0)| \exp \left(-\frac{\varepsilon}{n+1} p^{n+1}-\Im(\Omega) p\right) \\
& \times\left|\int_{-\infty}^{p} x \exp \left(\frac{\varepsilon}{n+1} x^{n+1}-\frac{x^{2}}{4}-i \Omega x\right) d x\right| \\
& \leq \frac{\alpha}{2}|G(0)| \exp \left(-\frac{\varepsilon}{n+1} p^{n+1}-\Im(\Omega) p\right) \\
& \times \int_{-\infty}^{p}|x| \exp \left(\frac{\varepsilon}{n+1} x^{n+1}-\frac{x^{2}}{4}+\Im(\Omega) x\right) d x=: f(p) .
\end{aligned}
$$

The function $f(p)$ is positive definite, and we are interested in its behavior for $p \rightarrow \infty$. By taking its derivative one finds that

$$
\frac{d f}{d p}(p)=\left(-\varepsilon p^{n}-\Im(\Omega)\right) f(p)+\frac{\alpha}{2}|G(0)| \cdot|p| e^{-p^{2} / 4} .
$$

Here, $\Omega$ is merely a parameter and can therefore be neglected in the prefactor in front of the first term in Eq. (25) for large values of $p$, and the second term tends rapidly to zero when its argument increases. This gives the relation

$$
\lim _{p \rightarrow \infty} \frac{d f}{d p}(p)=-\varepsilon \lim _{p \rightarrow \infty} p^{n} f(p)
$$

Since $f(p)$ is positive definite, $d f(p) / d p<0$ for large $p$, i.e., $f(p)$ decreases monotonically with $p$ when $p \rightarrow+\infty$. In this case there are only two options for the behavior of $f(p)$ at large $p$ : it can tend to some positive constant or to zero. First, assume that $\lim _{p \rightarrow \infty} f(p)=$ const $>0$. Then the limit in Eq. (26) equals $-\infty$ which contradicts the assumption we made and also the fact that $f(p)$ is positive definite. Therefore, $f(p)$ must go to zero for $p \rightarrow \infty$. In order to satisfy the positive 
definiteness of $f(p)$, its derivative also should go to zero for large $p$ which means that $f(p)$ tends faster to zero than $p^{n}$ tends to infinity. Since $f(p)$ is an upper bound for $|G(p)|$ and $n \geq 2$, it is clear that for large values of the argument $|G(p)|$ should decrease fast enough such that it is integrable. The integrand in the first term is also a continuous function. Since integration is a continuous operation, the integral is also continuous. Thus, $|G(p)|$ is a continuous function which decreases rapidly enough for $p \rightarrow \pm \infty$ such that it can be integrated, i.e., $\int_{-\infty}^{+\infty}|G(p)| d p<\infty$, which means that the solution

$$
\begin{aligned}
G(p)= & \frac{\alpha}{2} G(0) \exp \left(-\frac{\varepsilon}{n+1} p^{n+1}+i \Omega p\right) \\
& \times \int_{-\infty}^{p} x \exp \left(\frac{\varepsilon}{n+1} x^{n+1}-\frac{x^{2}}{4}-i \Omega x\right) d x
\end{aligned}
$$

has a Fourier transform, i.e., the Fourier analysis is consistent.

The next step is to find the dispersion relation which arises from the self-consistency condition for $p=0$. This yields

$$
1-\frac{\alpha}{2} \int_{-\infty}^{0} x \exp \left(\frac{\varepsilon}{n+1} x^{n+1}-\frac{x^{2}}{4}-i \Omega x\right) d x=0 .
$$

To the best of our knowledge, the above integral cannot be expressed in a closed form by using only elementary functions or the well-known special functions and is, therefore, rather inconvenient to work with. For this reason we make an expansion of the integrand in Eq. (28). Since $\varepsilon$ is proportional to the collision frequency, writing the exponential of $\varepsilon x^{n+1} /(n+1)$ as a series will help us determine the asymptotic behavior of the dispersion relation when the collision frequency tends to zero. At this point, one might be tempted to exchange summation and integration since all the integrals that emerge this way exist. However, such a term by term integration cannot be justified mathematically in this case. Since we are interested only in the asymptotic behavior $\varepsilon \rightarrow 0$, we apply the method of Borel summation, a simple description of which is given in Ref. 33 and the references therein. For that reason $\exp \left(\varepsilon x^{n+1} /(n+1)\right)$ is represented as

$$
\begin{aligned}
\exp \left(\frac{\varepsilon}{n+1} x^{n+1}\right)=1 & +\frac{\varepsilon}{n+1} x^{n+1} \\
& +\int_{0}^{\varepsilon} \frac{x^{2(n+1)}}{(n+1)^{2}}(\varepsilon-t) \exp \left(\frac{t}{n+1} x^{n+1}\right) d t .
\end{aligned}
$$

Further calculations will be facilitated by the relation

$$
\int_{-\infty}^{0} x^{r} e^{-x^{2} / 4-i \Omega x} d x=i^{r} \frac{d^{r}}{d \Omega^{r}} \int_{-\infty}^{0} e^{-x^{2} / 4-i \Omega x} d x=-i^{r+1} \frac{d^{r} Z(\Omega)}{d \Omega^{r}}
$$

which applies to all $r \in \mathbb{N}_{0}$, where $Z$ is the plasma dispersion function defined in Ref. 27. The above expression can be easily verified by induction when one takes into account that $Z(\Omega)=i \sqrt{\pi} e^{-\Omega^{2}}(1+\operatorname{erf}(i \Omega))$ and $Z^{\prime}(\Omega)=-2(1+\Omega Z(\Omega))$. Now, we estimate the difference, $D$, between the exact integral in Eq. (28) and its approximation achieved by taking only the first two terms on the right-hand side of Eq. (29) into account. A straightforward computation yields

$$
\begin{aligned}
D & =\left|\int_{-\infty}^{0} e^{-x^{2} / 4-i \Omega x} \frac{x^{2(n+1)}}{(n+1)^{2}} x \int_{0}^{\varepsilon}(\varepsilon-t) e^{t x^{n+1} /(n+1)} d t d x\right| \\
& \leq \int_{-\infty}^{0}\left|e^{-x^{2} / 4-i \Omega x}\right| \frac{x^{2(n+1)}}{(n+1)^{2}}|x| \int_{0}^{\varepsilon}(\varepsilon-t) e^{t x^{n+1} /(n+1)} d t d x .
\end{aligned}
$$

For $n$ even and $x \in(-\infty, 0]$ one can estimate the integral over $t$ from above as

$$
\int_{0}^{\varepsilon}(\varepsilon-t) \exp \left(\frac{t}{n+1} x^{n+1}\right) d t \leq \int_{0}^{\varepsilon}(\varepsilon-t) d t=\frac{1}{2} \varepsilon^{2} .
$$

This leads to an upper bound for $D$ given by

$$
D \leq \frac{\varepsilon^{2}}{2(n+1)^{2}} \int_{-\infty}^{0}|x| x^{2(n+1)} e^{-x^{2} / 4+\Im(\Omega) x} d x=: \varepsilon^{2} C_{n}(\Omega),
$$

where $C_{n}(\Omega)$ is a constant that depends on $n$ and $\Omega$ but not on $\varepsilon$ and is finite for every finite $\Omega$. Although $C_{n}(\Omega) \rightarrow \infty$ for $\Im(\Omega) \rightarrow-\infty$, setting a maximal damping rate above which modes are of no physical importance gives us a global upper bound for $C_{n}$ that is independent of $\Omega$ in that part of the complex plane. Therefore, by replacing the integral in Eq. (28) with $-2(1+\Omega Z(\Omega))+i^{n+1} \frac{\varepsilon}{n+1} d^{n+2} \frac{d \Omega^{n+2}}{} Z(\Omega)$, one only introduces an error of the order of $\varepsilon^{2}$, i.e., the dispersion relation reads

$1+\alpha+\alpha \Omega Z(\Omega)+i^{n+1} \frac{\varepsilon}{n+1} \alpha \frac{d^{n+1}}{d \Omega^{n+1}}(\Omega Z(\Omega))+O\left(\varepsilon^{2}\right)=0$.

From the above expression it is clear that in the limit $\varepsilon \rightarrow 0$, one recovers the well known Landau dispersion relation for a collisionless plasma. For the sake of completeness we also compute the first order contribution. Its exact form, however, depends on $n$. For a standard hyperdiffusion term $(n=4)$ the dispersion relation up to first order in $\varepsilon$ is

$$
\begin{aligned}
& 1+\alpha+\alpha \Omega Z(\Omega)+i \alpha \frac{\varepsilon}{5}\left[\left(-32 \Omega^{5}+224 \Omega^{3}-264 \Omega\right)\right. \\
& \left.\quad+\left(-32 \Omega^{6}+240 \Omega^{4}-360 \Omega^{2}+60\right) Z(\Omega)\right]+O\left(\varepsilon^{2}\right)=0 .
\end{aligned}
$$

The analysis in this part regarded so far only the Case-Van Kampen spectrum, i.e., we searched for solutions with a time dependence of $e^{-i \omega t}$. On the other hand, one could solve the initial value problem by using a Laplace transform with 
respect to time. For a Lenard-Bernstein collision operator, this results in the same dispersion relation as the one derived in Ref. 6. It is straightforward to analyze the linearized Vlasov equation with hyperdiffusion-like terms using such a Laplace transform. In this case, we found the same dispersion relation as in Eq. (28), i.e., the Case-Van Kampen spectrum coincides with the Landau solutions also when the dissipation term is of the form $-i \varepsilon\left(-i^{n} \partial^{n} g / \partial u^{n}\right)$, which is consistent with the results of our numerical analysis.

\section{B. Slab ITG modes}

In the following, we study the effect of hyperdiffusion terms on slab ITG modes which is slightly more complicated than for Langmuir waves, but the main idea remains the same. The linearized Vlasov equation is given in Eq. (14). In order to make the notation more convenient, we redefine the quantities there as follows:

$$
\begin{aligned}
\Omega & :=\frac{\omega}{\sqrt{2} k_{\|} \rho_{*}} ; \quad \kappa:=\frac{k_{y}}{\sqrt{2} k_{\|}} ; \quad u:=\frac{v_{\|}}{\sqrt{2}} ; \\
g(u) & :=f_{1, i}\left(\ldots, v_{\|}, \ldots\right) \quad \text { and } \quad g_{0}(u):=\sqrt{2} f_{0, i}\left(v_{\|}\right),
\end{aligned}
$$

where after the local approximation for the gradients, the equilibrium ion distribution function is a function only of the parallel velocity and is given by $e^{-v_{\|}^{2} / 2} / \sqrt{2 \pi}$. With this new notation and after adding a hyperdiffusion-like term Eq. (14) becomes

$$
\begin{aligned}
\Omega g= & u g+\frac{\tau}{\sqrt{\pi}}\left(\frac{\kappa}{L_{T}} u^{2}+u+\frac{\kappa}{L_{d}}-\frac{\kappa}{2 L_{T}}\right) e^{-u^{2}} \int_{-\infty}^{+\infty} g\left(u^{\prime}\right) d u^{\prime} \\
& -i \varepsilon\left(i^{n} \frac{\partial^{n} g}{\partial u^{n}}\right) .
\end{aligned}
$$

After Fourier transforming Eq. (37) with respect to $u$, one arrives at a first-order ordinary differential equation which reads

$$
\frac{d G}{d p}(p)+\left(\varepsilon p^{n}-i \Omega\right) G(p)=\tau G(0)\left(\frac{i \kappa}{4 L_{T}} p^{2}+\frac{1}{2} p-\frac{i \kappa}{L_{d}}\right) e^{-p^{2} / 4}
$$

and is of the same type as Eq. (22). The differential operator on the left-hand side of Eq. (38) is the same as that in Eq. (22) and, therefore, has the same kernel. The only difference is the right-hand side. However, this difference is only quantitative and not qualitative because in both cases the righthand side is a Schwartz function. The general solution of the above differential equation reads

$$
\begin{aligned}
G(p)= & C \exp \left(-\frac{\varepsilon}{n+1} p^{n+1}+i \Omega p\right) \\
& +\tau G(0) \exp \left(-\frac{\varepsilon}{n+1} p^{n+1}+i \Omega p\right) \\
& \times \int_{a}^{p}\left(\frac{i \kappa}{4 L_{T}} x^{2}+\frac{1}{2} x-\frac{i \kappa}{L_{d}}\right) \exp \left(\frac{\varepsilon}{n+1} x^{n+1}-\frac{x^{2}}{4}-i \Omega x\right) d x,
\end{aligned}
$$

where $C$ and $a$ are arbitrary constants that are fixed by demanding that $G(p)$ should have a Fourier transform. This leads to $C=0$ and $a=-\infty$ as for electrostatic Langmuir waves. Derivation of the dispersion relation requires setting $p=0$. The resulting integral is again rather sophisticated and, to the best of our knowledge, cannot be expressed in a closed form using elementary functions or the well-known special functions. Therefore, we proceed as before and apply the method of Borel summation to the infinite series that the integrand can be represented as. After completely analogous calculations one derives the dispersion relation up to order $\varepsilon$ which reads

$$
\begin{aligned}
& 1+\frac{1}{\tau}+\frac{\kappa}{L_{T}} \Omega+Z(\Omega)\left(\frac{\kappa}{L_{T}} \Omega^{2}+\Omega+\frac{\kappa}{L_{n}}-\frac{\kappa}{2 L_{T}}\right) \\
& +i^{n+1} \frac{\varepsilon}{n+1} \frac{d^{n+1}}{d \Omega^{n+1}}\left[\frac{\kappa}{L_{T}} \Omega+Z(\Omega)\left(\frac{\kappa}{L_{T}} \Omega^{2}+\Omega+\frac{\kappa}{L_{n}}-\frac{\kappa}{2 L_{T}}\right)\right] \\
& +O\left(\varepsilon^{2}\right)=0 .
\end{aligned}
$$

By taking the limit $\varepsilon \rightarrow 0$, one arrives at the Landau dispersion relation for collisionless slab ITG modes.

As a last point we note that solving the initial value problem via a Laplace transform in time leads again to the same solutions as those composing the Case-Van Kampen spectrum.

In conclusion, velocity space hyperdiffusion effectively captures some important physical properties of the genuine collision operator as also the Lenard-Bernstein operator does. However, in contrast to the latter, hyperdiffusion in velocity space conserves not only particle number, but it implies momentum (for $n>2$ also energy) conservation, too.

\section{SUMMARY}

We have investigated the linearized problem of electrostatic plasma oscillations numerically and analytically. In the collisionless case, we showed that formulating the problem in the language of operator theory provides an elegant explanation of the continuous and discrete parts of the Case-Van Kampen spectrum. Additionally a sufficient condition for the existence of the former was given. For the slab ITG modes, the discrete part of the collisionless spectrum is related to possible instabilities and drift waves that arise in the presence of background gradients. A numerical investigation of collisionless Langmuir waves and slab ITG modes showed that CaseVan Kampen modes tend to gather above the least damped Landau solutions but, nevertheless, all the modes are needed in order to reproduce the effect of Landau damping.

The collisional system was treated first numerically using different discretization schemes. A finite difference scheme on an equidistant velocity grid, which is quite common in numerical studies, yielded a large number of nonphysical modes with small damping rates. In extreme cases, some of those artificial modes are less damped even than the least damped Landau solutions which could potentially make the analysis more difficult if the latter are unknown. Note that such numerical modes do not influence the temporal 
evolution of moments of the distribution function, e.g., of the electrostatic potential. However, discretization via expansion of the perturbation into a basis of Hermite polynomials multiplied by a Gauss function leads to a spectrum with more identifiable Landau solutions. Moreover, all nonphysical modes present in this case are more strongly damped than the physical Landau solutions. In contrast to the finite difference scheme, the Hermite representation produces spectra wherein all modes with small damping rates have a straightforward physical interpretation. This may prove beneficial for the analysis of numerical simulations of nonlinear models. In this sense, a truncated Hermite representation is superior to a finite difference scheme on an equidistant velocity space grid.

From our numerical analysis, it became apparent that the qualitative change of the eigenvalue spectrum for any infinitesimal collision frequency is due only to the second derivative of the perturbation with respect to velocity. This we then investigated also analytically and showed that any even power of velocity space hyperdiffusion leads to Case-Van Kampen eigenvalues that match the collisionless Landau solutions when the hyperdiffusivity tends to zero. Although such hyperdiffusion terms are used primarily for purely numerical reasons, our analysis shows that they correctly reproduce important physical effects, e.g., Landau damping.

\section{ACKNOWLEDGMENTS}

The first author of the paper is grateful to Semjon Wugalter and Omar Maj for valuable discussions. Further, very insightful discussions with John Krommes are acknowledged. The research leading to these results has received funding from the European Research Council under the European Union's Seventh Framework Programme (FP7/20072013)/ERC Grant Agreement No. 277870.

${ }^{1}$ L. Landau, J. Phys. USSR 10, 25 (1946).

${ }^{2}$ J. H. Malmberg and C. B. Wharton, Phys. Rev. Lett. 13, 184 (1964).

${ }^{3}$ N. G. Van Kampen, Physica 21, 949 (1955).
${ }^{4}$ A. Lenard and I. B. Bernstein, Phys. Rev. 112, 1456 (1958).

${ }^{5}$ C. S. Ng, A. Bhattacharjee, and F. Skiff, Phys. Rev. Lett. 83, 1974 (1999).

${ }^{6}$ R. W. Short and A. Simon, Phys. Plasmas 9, 3245 (2002).

${ }^{7}$ F. Jenko, W. Dorland, M. Kotschenreuther, and B. N. Rogers, Phys. Plasmas 7, 1904 (2000).

${ }^{8}$ M. Kammerer, F. Merz, and F. Jenko, Phys. Plasmas 15, 052102 (2008).

${ }^{9}$ E. A. Belli and J. Candy, Phys. Plasmas 17, 112314 (2010).

${ }^{10}$ D. R. Hatch, P. W. Terry, F. Jenko, F. Merz, M. J. Plueschel, W. M. Nevins, and E. Wang, Phys. Plasmas 18, 055706 (2011).

${ }^{11}$ F. Merz, C. Kowitz, E. Romero, J. E. Roman, and F. Jenko, Comput. Phys. Commun. 183, 922 (2012).

${ }^{12}$ T. Dannert and F. Jenko, Phys. Plasmas 12, 072309 (2005).

${ }^{13}$ G. M. Staebler, J. E. Kinsey, and R. E. Waltz, Phys. Plasmas 12, 102508 (2005).

${ }^{14}$ C. Bourdelle et al., Phys. Plasmas 14, 112501 (2007).

${ }^{15}$ F. Merz and F. Jenko, Phys. Rev. Lett. 100, 035005 (2008).

${ }^{16}$ F. Merz and F. Jenko, Nucl. Fusion 50, 054005 (2010).

${ }^{17}$ D. R. Hatch, P. W. Terry, F. Jenko, F. Merz, and W. M. Nevins, Phys. Rev. Lett. 106, 115003 (2011).

${ }^{18}$ P. W. Terry, D. A. Baver, and S. Gupta, Phys. Plasmas 13, 022307 (2006).

${ }^{19}$ B. Teaca, A. B. Navarro, F. Jenko, S. Brunner, and L. Villard, Phys. Rev. Lett. 109, 235003 (2012).

${ }^{20}$ Methods of Modern Mathematical Physics, IV: Analysis of Operators, edited by M. Reed and B. Simon (Academic, 1978).

${ }^{21}$ B. Wu, J. Phys. A: Math. Gen. 32, 5835 (1999).

${ }^{22}$ Theoretical Methods in Plasma Physics, edited by N. G. Van Kampen and B. U. Felderhof (North-Holland, Amsterdam, 1967).

${ }^{23}$ K. M. Case, Ann. Phys. (N.Y.) 7, 349 (1959).

${ }^{24}$ See http://www.theorie.physik.uni-muenchen.de/TMP/theses/thesis bratanov.pdf.

${ }^{25}$ B. Coppi, M. N. Rosenbluth, and R. Z. Sagdeev, Phys. Fluids 10, 582 (1967).

${ }^{26}$ A. M. Dimits, G. Bateman, M. A. Beer, B. I. Cohen, W. Dorland, G. W. Hammett, C. Kim, J. E. Kinsey, M. Kotschenreuther, A. H. Kritz, L. L. Lao, J. Mandrekas, W. M. Nevins, S. E. Parker, A. J. Redd, D. E. Shumaker, R. Sydora, and J. Weiland, Phys. Plasmas 7, 969 (2000).

${ }^{27}$ The Plasma Dispersion Function, edited by B. D. Fried and S. D. Conte (Academic, London, 1961).

${ }^{28}$ J. P. Holloway and J. J. Dorning, Phys. Rev. A 44, 3856-3868 (1991).

${ }^{29}$ Formulas and Theorems for the Special Functions of Mathematical Physics, edited by W. Magnus, F. Oberhettinger, R. P. Soni (Springer, Berlin/Heidelberg/New York, 1966).

${ }^{30}$ F. Skiff, S. De Souza-Machado, W. A. Noonan, A. Case, and T. N. Good, Phys. Rev. Lett. 81, 5820 (1998).

${ }^{31}$ J. Parker, private communication (2012)

${ }^{32}$ M. J. Pueschel, T. Dannert, and F. Jenko, Comput. Phys. Commun. 181, 1428-1437 (2010).

${ }^{33}$ M. Flory, R. C. Helling and C. Sluka, arXiv:1201.2714v2 [math-ph]. 